\title{
miR-503 Is Involved in the Protective Effect of Phase II Enzyme Inducer (CPDT) in Diabetic Cardiomyopathy via Nrf2/ARE Signaling Pathway
}

\author{
Ying Miao, Qin Wan, Xiaoyu Liu, Yu Wang, Yi Luo, Dan Liu, Nengbo Lin, \\ Honggang Zhou, and Jiyuan Zhong \\ Department of Endocrinology, The Affiliated Hospital of Southwest Medical University, Luzhou 646000, China \\ Correspondence should be addressed to Qin Wan; wanqin3@163.com
}

Received 22 August 2017; Revised 8 October 2017; Accepted 25 October 2017; Published 18 December 2017

Academic Editor: Osamu Handa

Copyright (C) 2017 Ying Miao et al. This is an open access article distributed under the Creative Commons Attribution License, which permits unrestricted use, distribution, and reproduction in any medium, provided the original work is properly cited.

\begin{abstract}
Diabetic cardiomyopathy (DCM) is a common heart disease. The Phase II enzyme inducer (CPDT) is a complex enzyme that promotes the expression of antioxidant enzymes through activating nuclear factor erythroid 2-related factor 2 (Nrf2); these compounds have been shown to protect against oxidative stress. However, whether these compounds have similar protective effects in DCM still remains unclear. The purpose of this study is to investigate the protective effects and potential mechanism of CPDT in diabetic cardiomyopathy. In the results, firstly, compared with control rats, myocardial cell size, left ventricular mass index, and myocardial apoptosis index were increased, miR-503 was increased, and Nrf2, malondialdehyde (MDA), and heme oxygenase 1 (HO-1) were decreased in diabetic cardiomyopathy rats. Furthermore, compared with diabetic cardiomyopathy rats, these above parameters show the opposite change in CPDT treatment rats. In addition, the bioinformatics and luciferase reporter assay demonstrated that Nrf2 is a direct target of miR-503. Finally, the miR-503 could also regulate Nrf2 in the myocardial cells. Therefore, miR-503 is involved in the protective effect of CPDT in diabetic cardiomyopathy via Nrf2/ARE signaling pathway; miR503 and Nrf2 may be a promising therapeutic target for the management of diabetic cardiomyopathy.
\end{abstract}

\section{Background}

Diabetic cardiomyopathy (DCM) is the main cardiovascular complication, which occurs in approximately $60 \%$ of patients with well-controlled diabetes, resulting in systolic dysfunction with diastolic dysfunction, which is an independent risk for any vascular disease or hypertension [1]. An increasing body of evidences implicate hyperglycemia, lipotoxicity, and mitochondrial uncoupling in contributing to cardiac inflammation, which play an important role in the pathogenesis and progression of diabetic cardiomyopathy $[2,3]$. In this process, cytoplasmic calcium was increased, and then, triggering mitochondrial changes, the production of reactive oxygen species (ROS) was increased, and activating ROS levels leads to oxidative damage in diabetic cardiomyopathy [4].

Phase II enzyme inducer such as 5,6-dihydrocyclopenta1,2-dithiole-3-thione (CPDT), 3H-1,2-dithiole-3-thione (D3T), and tert-butylhydroquinone (t-BHQ) were initially synthesized as a protective agent for a wide variety of chemical carcinogens [5]. This protective effect depends on the nuclear factor erythroid 2-related factor 2 (Nrf2)/antioxidant response element (ARE) pathway by increasing the activity of the second detoxification enzymes, including antioxidant enzymes such as malondialdehyde (MDA) and heme oxygenase 1 (HO-1) [6]. In addition, recently, the Phase II enzyme inducer was shown to bring neuroprotective effect against mitochondrial stress induced by mitochondrial complex II inhibitor 3-nitropropionic acid, by improving neurons survival, and attenuate the production of ROS in response to lipopolysaccharide treatment of cells [7]. However, there is no evidence to show these agents have a protective effect on diabetic cardiomyopathy.

MicroRNAs are endogenous, small noncoding RNAs, approximately 19-22 nucleotides in length, with complementary sequences in the $3^{\prime}$-untranslated regions ( $3^{\prime}$-UTRs) of downstream mRNAs to promote its degradation or inhibit 
translation process [8,9]. Extensive studies have shown that microRNAs are involved in a wide variety of biological processes, including cell proliferation, differentiation, metastasis, apoptosis, and immune responses, also used as prognostic markers genes related to the development and progression of cardiovascular disease [10, 11]. For miR503 , some studies have showed that miR-503 was increased in diabetic muscles and oppositely correlated with cdc25 protein expression; in addition, miR-503 was also increased in myocardial microvascular endothelial cells from type 2 diabetic Goto-Kakizaki (GK) rats [12]; finally, compared with normal healthy controls, miR-503 was also increased in type 2 diabetes patients [13]. Therefore, miR-503 plays important role in diabetes; however, the role and mechanism of miR-503 in DCM remain unclear.

The aim of this study was to elucidate the essentially protective effect of Phase II enzyme inducer (CPDT) and the underlying mechanisms in diabetic cardiomyopathy. In this study, diabetic cardiomyopathy model in rats was duplicated, and Phase II enzyme inducer CPDT, as intervention agent, was used to detect relevant indicators, such as miR503 and Nrf2/ARE signaling pathway, including Nrf2, HO1 and MDA, to investigate whether the Phase II enzyme inducer (CPDT) reduces myocardial cell apoptosis, reduces the occurrence and development of diabetic cardiomyopathy through miR-503 and Nrf2/ARE signaling pathway, and provides new therapeutic targets for diabetic cardiomyopathy.

\section{Material and Methods}

2.1. Ethics Statement. The study was approved by the ethics committee of the Affiliated Hospital of Southwest Medical University, and all experimental procedures were approved by the animal care and use committee of the Affiliated Hospital of Southwest Medical University. The study was performed according to the recommendations of the guide for the care and use of laboratory animals.

\subsection{Animals and Establishment of Diabetic Model and Treat-} ment. The healthy male Wistar rats $(180 \sim 220 \mathrm{~g})$ were purchased from Southwest Medical University and housed at a temperature of $22 \pm 1^{\circ} \mathrm{C}$ and humidity of $55 \pm 5 \%$. The rats were divided randomly into control group (C) and diabetes mellitus (DM) group. Rat diabetic model was established according to previous report [14] with slight modification. A high-fat diet was prepared with $20 \%$ lard, $5 \%$ cholesterol, $5 \%$ sucrose, $5 \%$ glucose, and $6 \%$ salt and emulsified in $20 \%$ Tween- 80 and $30 \%$ propylene glycol with distilled water. Rats in the DM group were fed with $2 \mathrm{ml}$ prepared high-fat diet per day. $30 \mathrm{mg} / \mathrm{kg} / \mathrm{d}$ of streptozotocin (STZ) (Sigma, USA) in $0.1 \mathrm{M}$ citrate buffer solution was injected intraperitoneally in diabetic rats for 3 consecutive days. $72 \mathrm{~h}$ later, fasting blood glucose levels were measured, and blood glucose levels $>16.7 \mathrm{mmol} / \mathrm{l}$ diabetes was regarded as diabetic model established successfully. The model rats were randomly divided into diabetic group and intervention group with 10 rats in each group. All the rats were fed with high sugar and high-fat diet, and the intervention group was given Phase II enzyme inducer (CPDT) (Sigma, St. Louis,
MO, USA) $500 \mathrm{umol} / \mathrm{kg} /$ day for 14 days. All the rats were fed with high sugar and high-fat diet for 8 weeks.

2.3. Light and Electron Microscope Examination. For electron microscope examination, the heart was cut into small pieces and prefixed in $2.5 \%$ glutaraldehyde $(0.2 \mathrm{M}$ cacodylate buffer, $\mathrm{pH}$ 7.4) for $4 \mathrm{~h}$, postfixed in $1 \%$ buffered sodium tetroxide for $1 \mathrm{~h}$, and embedded in epoxy resin by routine methods. Ultrathin sections were examined using a JEM-1200 EX electron microscope (JEOL Ltd., Tokyo, Japan).

2.4. HE Staining. Tissues were fixed in $4 \%$ paraformaldehyde. The samples were embedded in paraffin, cut into $5 \mu \mathrm{m}$ thick sections and stained with hematoxylin-eosin (HE) for histological and collagen analysis, according to the manufacturers' protocols.

2.5. TUNEL Staining. Tissues were fixed in $4 \%$ paraformaldehyde tissue, and TUNEL fluorescence FITC kit (Roche, USA) was utilized for cell apoptosis assay as previous report [14]. Terminal deoxynucleotidyl transferase dUTP nick end labeling staining was used to detect DNA fragmentation (green fluorescence) with the nuclei stained with DAPI (blue fluorescence). Images were captured through fluorescence microscopy (Nikon Co., Japan).

2.6. RNA Extraction and $q R T-P C R$. Total RNA was extracted with the RNeasy plus mini kit (Qiagen, USA). Total RNA concentrations were determined by NanoVue plus (GE Healthcore, Piscataway, NJ, USA). miR-503 was detected with the TaqMan miRNA assays and U6 as an internal control. Nrf2, HO-1, and MDA were detected by qRT-PCR with the SYBR Premix Ex Taq II kit (TaKaRa, Dalian, China) and the Applied Biosystems ABI Prism 7500 HT sequence detection system. The primers of relative gene were as follows:

\section{Nrf2: $\quad 5^{\prime}$-ACGTGGCTAAGAATGTCATC-3' (forward) and $5^{\prime}$-CTGGTAGGCGATGTCCTTA- $3^{\prime}$ (reverse) \\ HO-1: $5^{\prime}$-ACTCGAACGACTCTGATGATGT-3' (forward) and $5^{\prime}$-GTCAGGTCTGCGAAACTT- CTTA-3' (reverse) \\ MDA: $5^{\prime}$-TCTTCACAAATCCTCCCC-3' (forward) and $5^{\prime}$-TGGATTAAAAGGACTTGG-3' (reverse).}

The expression of mRNA or microRNA was evaluated based on the threshold cycle (Ct) as $n=2^{-\Delta \Delta \mathrm{Ct}}$, where $\Delta \mathrm{Ct}=\mathrm{Ct}$ related $\mathrm{mRNA}-\mathrm{Ct}$ reference and $\Delta \Delta \mathrm{Ct}=$ $\Delta \mathrm{Cq}$ experimental $-\Delta \mathrm{Ct}$ control.

2.7. Western Blotting. The protein samples were collected and protein samples of $100 \mu \mathrm{g}$ each line were loaded on a $10 \%$ SDS-PAGE. Protein was transferred to PVDF membrane, which was subsequently blocked by $5 \%$ nonfat milk dissolved in PBS for $2 \mathrm{~h}$; then, PVDF membrane was incubated with primary antibodies at $4^{\circ} \mathrm{C}$ overnight and then incubated with appropriate HRP-conjugated secondary antibodies at room temperature for $1 \mathrm{~h}$. Finally, the bands were visualized with 
the chemiluminescence method and Western blotting bands were quantified using the Odyssey Infrared Imaging System (LI-COR, Lincoln, NE, USA). The Nrf2, HO-1, MDA, and $\beta$-actin antibody and HRP-conjugated secondary antibodies were purchased from Santa Cruz Biotechnology.

2.8. Prediction of Target Gene. Database of miRanda, TargetScan, and PicTar was used to predict the potential targets gene of miR-503. Among those target genes, nuclear factor erythroid 2-related factor 2 (Nrf2) had a binding site in the $3^{\prime}$-UTR of miR-503.

2.9. Luciferase Reporter Assays. The wild-type- (WT-) Nrf2 $3^{\prime}$-UTRs and the mutated-Nrf2 $3^{\prime}$-UTRs were synthesized by Sangon Biotech Co., Ltd. (Shanghai, China) and amplified by PCR. The forward primer was 5'-CCGCTCGAGAGGATCACTGAGGAAGGGGAAGTG- $3^{\prime}$ and the reverse primer was $5^{\prime}$-ATAAGAATGCGGCCGCGCCTTGTACTACACATGTGTGACTGATC-3'.

The wild-type (WT) and mutated (Mut) exon of Nrf2 were inserted into the downstream of firefly luciferase reporter gene in the psiCHECK-2 vector. The constructed luciferase reporters were called psiCHECK-2-Nrf2-3'-UTRWT and psiCHECK-2-Nrf2-3'-UTR-mut. For luciferase assay, The human embryonic kidney (HEK293T) 293 cells were seeded into 24 -well plates, and NC mimics (empty vector) and miR-503 mimics (stable miR-503-overexpressing) were cotransfected with constructed reporter plasmids (0.2 ug) into HEK293T cells by Effectene transfection regents (Qiagen). After 48 hours, the luciferase activities were measured using the Clarity ${ }^{\mathrm{TM}}$ Luminescence Microplate Reader.

2.10. Primary Isolation and Culture of Myocardial Cells. Primary myocardial cells were from the hearts of 1-3-dayold Wistar rats. Briefly, cardiac tissues were separated and digested by pancreatin, the isolated cells were cultured at $37^{\circ} \mathrm{C}, 5 \% \mathrm{CO} 2$, and resuspended in Dulbecco's modified Eagle's medium (Hyclone, Logan, UT, USA) containing 10\% fetal bovine serum (Hyclone); then, myocardial cells were purified through differential plating and 0.1 mM 5-bromo-2deoxyuridine was used to deplete nonmyocardial cells.

2.11. Transfection of Relative MicroRNA. Synthetic miR-503 mimic and inhibitor were generated and purchased from Sangon Biotech Co., Ltd. (Shanghai, China), which had ability of upregulation and downregulation of miR-503. The mimic and inhibitor were transfected into myocardial cells with lipofectamine 2000 (Invitrogen, USA) to induce upregulation and downregulation of miR-503 in the myocardial cells according to manufacturer's instructions.

2.12. Statistical Analysis. Data were presented as mean values and standard deviation. The independent-samples $t$-test or the analysis of variance (ANOVA) was used to identify differences among all groups (SPSS, USA). The $P$ value $<0.05$ was considered to be statistically significance.

\section{Results}

3.1. Heart Parameters in Streptozotocin- (STZ-) Induced Diabetes and Treatment Rats. After the STZ was used to induce diabetes mellitus (DM), diabetic cardiomyopathy (DCM) was further established, DCM rats were treated with Phase II enzyme inducer (CPDT), and relevant information was detected and analyzed. The results showed that, compared with control rats, fasting blood glucose was significantly increased and body weight was significantly increased in diabetes and treatment rats, and there was no statistical difference in fasting blood glucose and body weight between diabetes and treatment rats (Figures 1(a) and 1(b)). In addition, compared with control rats, total heart weight, left ventricular weight, heart to body ratio, and left ventricular mass index were significantly increased in diabetes rats; however, compared with diabetes rats, these parameters were significantly improved in treatment rats (Figures $1(\mathrm{c})-1(\mathrm{f})$ ).

3.2. Cardiopathology in Streptozotocin- (STZ-) Induced Diabetes and Treatment Rats. The animal model of diabetic cardiomyopathy was established, diabetic cardiomyopathy rats were treated with Phase II enzyme inducer (CPDT), and cardiopathology was detected and analyzed. The results from HE staining showed that, compared with control rats, myocardial hypertrophy and irregular arrangement myocardial cell were increased, myocardial cell nucleus was enlarged, the width of the intercellular space was widened, and the longitudinal connection between myocardial cells was decreased in diabetes rats; however, compared with diabetes rats, these parameters were significantly improved in treatment rats (Figures 2(a)-2(c)). The results from electron microscope examination showed that the cardiac fibers with regular arrays and the mitochondria were typically intact in control rats; however, the mitochondria and the myofibrils were swollen and disrupted in diabetes rats; however, these parameters were significantly improved in treatment rats (Figure 2(d)). The results from TUNEL staining showed that, compared with control rats, the apoptosis level was significantly increased in diabetes rats; however, compared with diabetes rats, the apoptosis level was significantly decreased in treatment rats (Figures 3(g) and 3(h)).

3.3. The Expression of miR-503, Nrf2, and Downstream Medium Level in Streptozotocin- (STZ-) Induced Diabetes and Treatment Rats. The diabetic cardiomyopathy was established, diabetic cardiomyopathy rats were treated with Phase II enzyme inducer (CPDT), and the miR-503, Nrf2, and downstream medium level were detected and analyzed. The result showed that, compared with control rats, miR-503 was significantly increased, and Nrf2, MDA, and HO-1 were significantly decreased in diabetes rats; however, compared with diabetes rats, miR-503 was significantly decreased, and $\mathrm{Nrf2}, \mathrm{MDA}$, and $\mathrm{HO}-1$ were significantly increased in treatment rats (Figures 3(a)-3(f)).

3.4. The Nrf2 Was the Target Gene of miR-503. According to the prediction analysis of the TargetScan, PicTar, and miRanda data, Nrf2 has a putative binding site in the $3^{\prime}$-UTR 


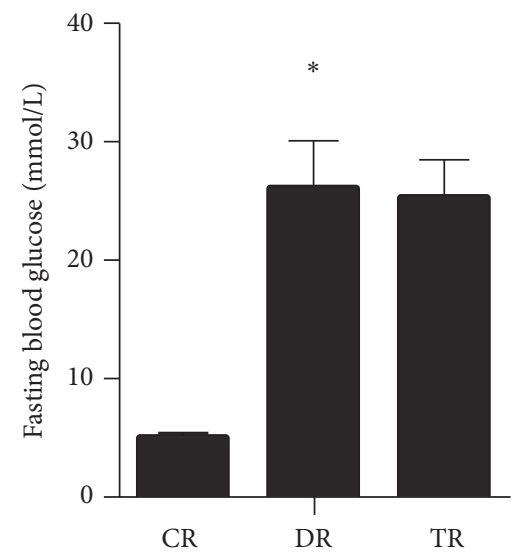

(a)

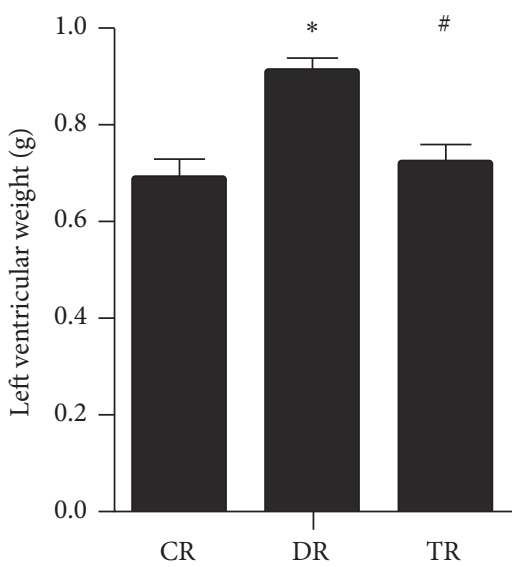

(d)

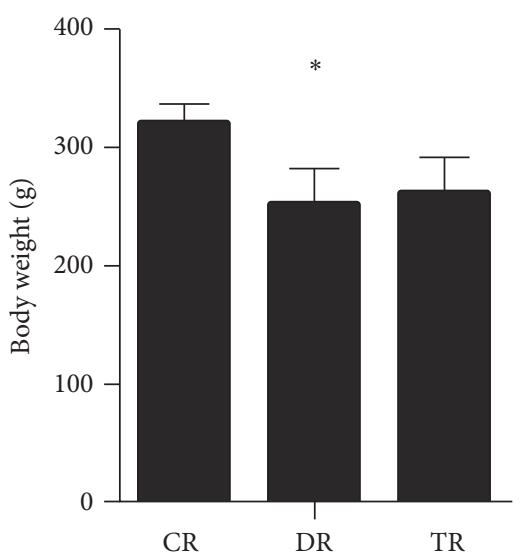

(b)

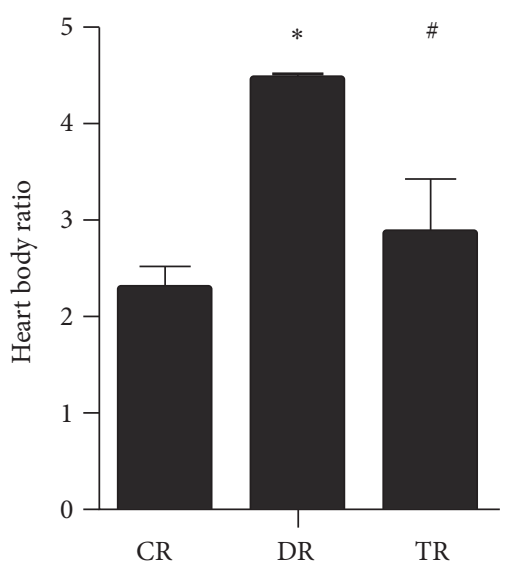

(e)

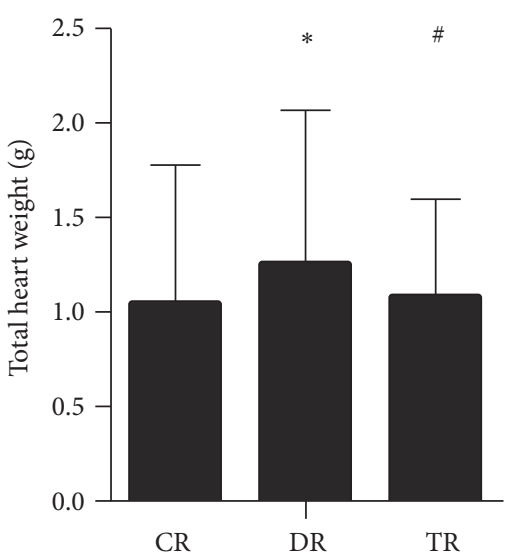

(c)

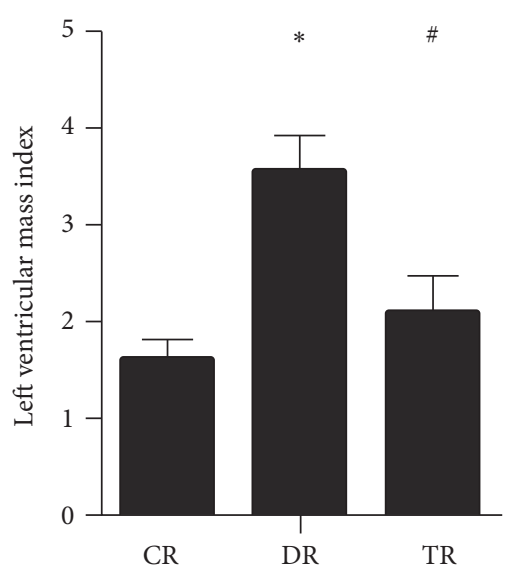

(f)

FIGURE 1: Fasting blood glucose, body weight, and cardiac dysfunction in streptozotocin- (STZ-) induced diabetes and treatment rats; CR: control rats; DR: diabetes rats; TR: treatment rats; $(\mathrm{a}-\mathrm{b})$ fasting blood glucose and body weight; (c-f) each weight's related indicators; $*$ : compared with control rats, $P$ value $<0.05$ was considered to be statistically significant; \#: compared with diabetes rats, $P$ value $<0.05$ was considered to be statistically significant.

of miR-503 and was speculated as the target gene of miR-503 (Figure 4(a)). The dual-luciferase reporter assay method was used to further investigate whether miR-503 directly targets Nrf2. The results showed that miR-503 suppressed luciferase activity through $3^{\prime}$-UTR of wild-type (WT) HMGB1 (Figure $4(\mathrm{~b}))$. In addition, the activity change of fluorescence of miR-503 containing the mutated $3^{\prime}$-UTR site or the control group lacking an Nrf2 $3^{\prime}$-UTR sequence had no statistically significant difference (Figure 4(c)).

3.5. miR-503 Regulated the Expression of Nrf2 in Myocardial Cells. After mimic and inhibitor were transfected into myocardial cells, the miR-503, Nrf2, and downstream medium level were detected and analyzed. The results showed that, compared with control, the intracellular miR-503 was increased by miR-503 mimics and decreased by miR- 503 inhibitor (Figure 5(a)). In addition, the expression of Nrf2 was decreased by miR-503 mimics and increased by inhibitor (Figures 5(b)-5(d)).

\section{Discussion}

Diabetic cardiomyopathy (DCM) is a common structural abnormality caused by myocardial cell injury in diabetes, leading to left ventricular hypertrophy and diastolic or systolic dysfunction [15]. Recently, numerous studies have reported that DCM is a kind of independent pathophysiology, and the main clinical manifestations are congestive heart failure due to cardiac diastolic and systolic dysfunction [16, 17]. Nowadays, the pathological mechanism of this disease still remains unclear. In addition, treatment for this disease is not ideal and it is difficult to prevent and treat this disease, ultimately leading to heart failure, arrhythmia, and cardiogenic shock, one of the important causes of sudden death [18]. In this study, we found that miR-503 is involved in the progress of DCM, and Phase II enzyme inducer (CPDT) could reverse the impaired structure and function, reduce myocardial apoptosis, relieve the occurrence and development of DCM through miR-503 and Nrf2/ARE signaling pathway, and provide new therapeutic targets for DCM. 


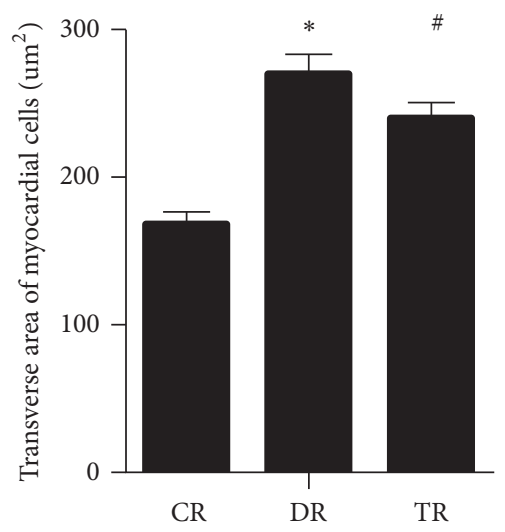

(a)

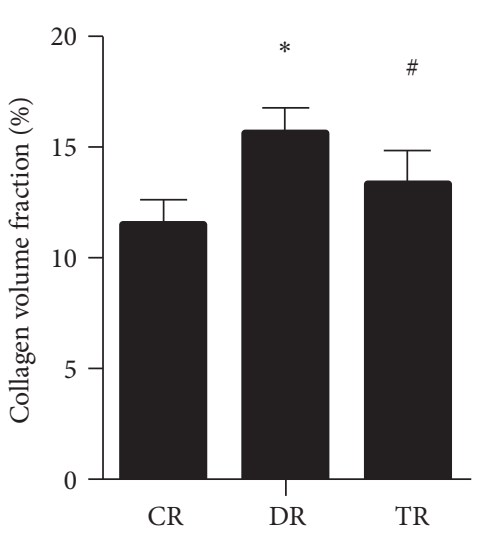

(b)
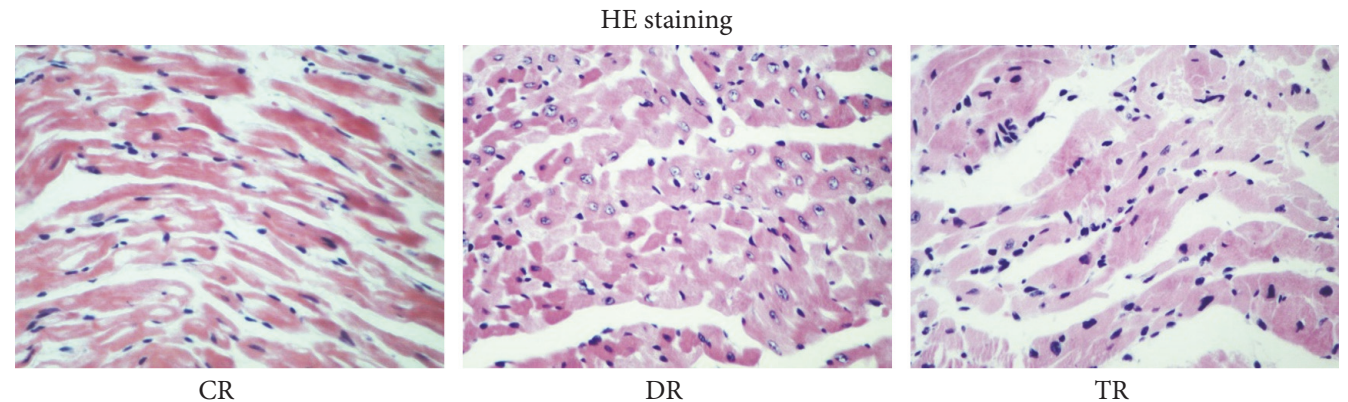

TR

(c)

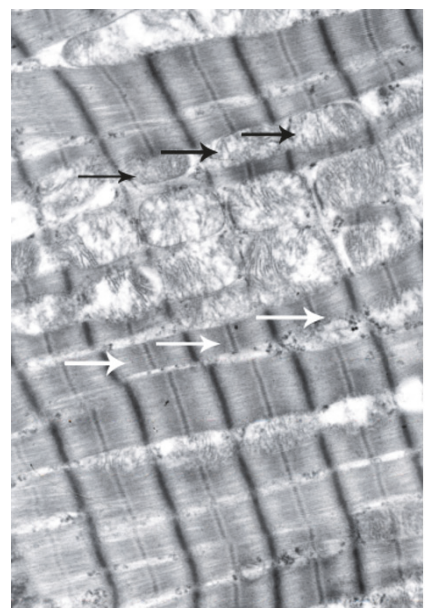

CR

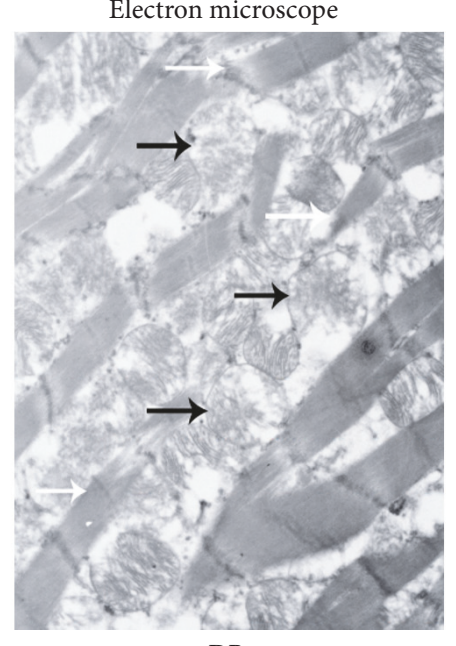

DR

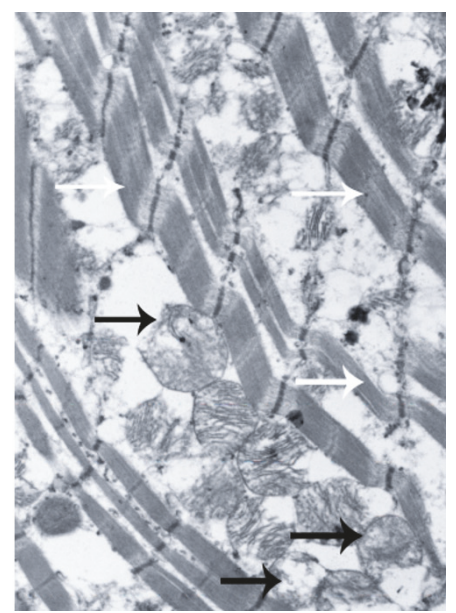

TR

(d)

FIGURE 2: Cardiopathology in streptozotocin- (STZ-) induced diabetes and treatment rats; CR: control rats; DR: diabetes rats; TR: treatment rats. (a) Transverse area of myocardial cells; (b) collagen volume fraction OF Myocardial cell; (c) HE staining ( $\times 400$ ); (d) electron microscope $(\times 10000$, the white arrow: cardiac fibers, and the black arrow: mitochondria); *: compared with control rats, $P$ value $<0.05$ was considered to be statistically significant; \#: compared with diabetes rats, $P$ value $<0.05$ was considered to be statistically significant.

Some studies have showed that the changes of tissue structure and function, oxidative stress, myocardial apoptosis, and necrosis are the important factors leading to occurrence and development of DCM $[19,20]$. In the early DCM, myocardial apoptosis and necrosis occurred, oxidative stress was increased, and the corresponding signal transduction pathway and gene expression were abnormal; thereby the programmed cell death was initiated [21]. In addition, some studies have also showed that in the development stage of DCM, during high blood glucose, the collagen glycosylation, degradation, and endocardial collagen deposition happened, the death of myocardial cells gradually is replaced, eventually, 


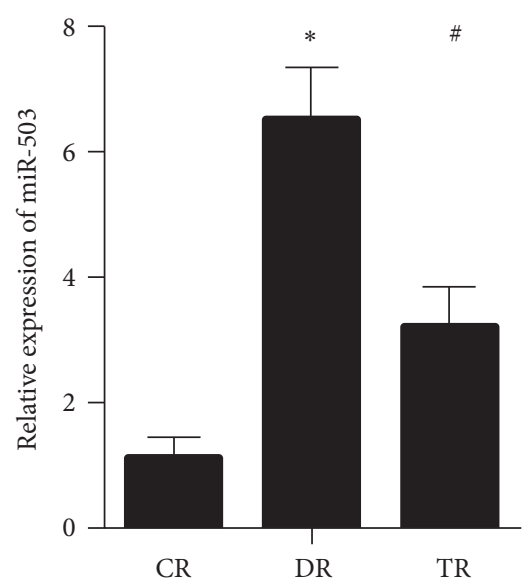

(a)

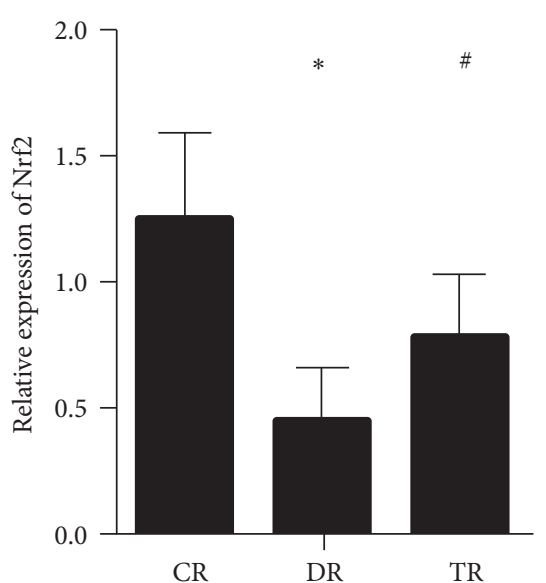

(b)

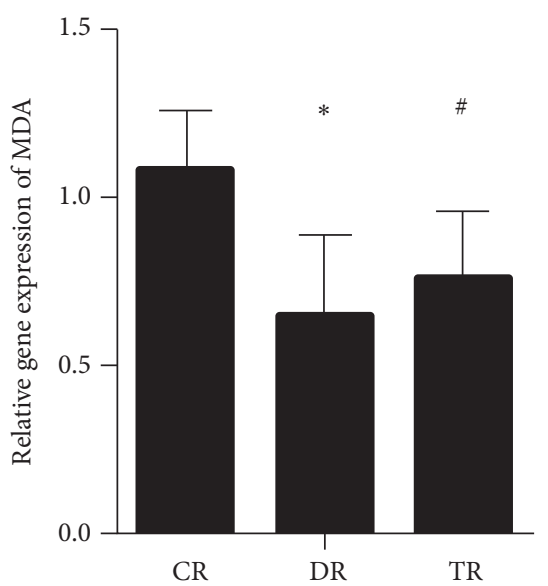

(c)

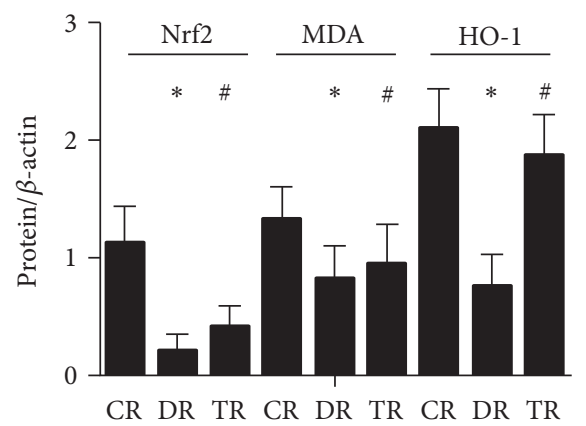

(f)

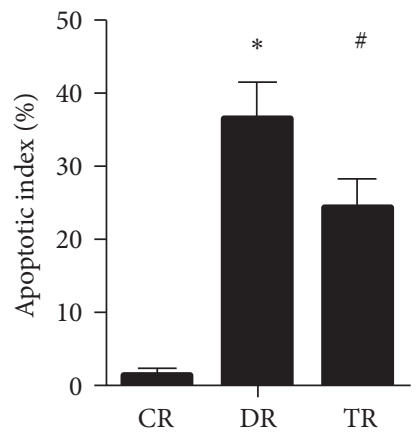

(g)

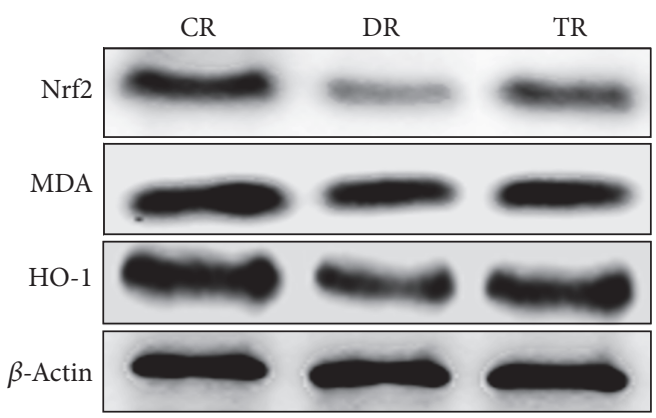

(e)

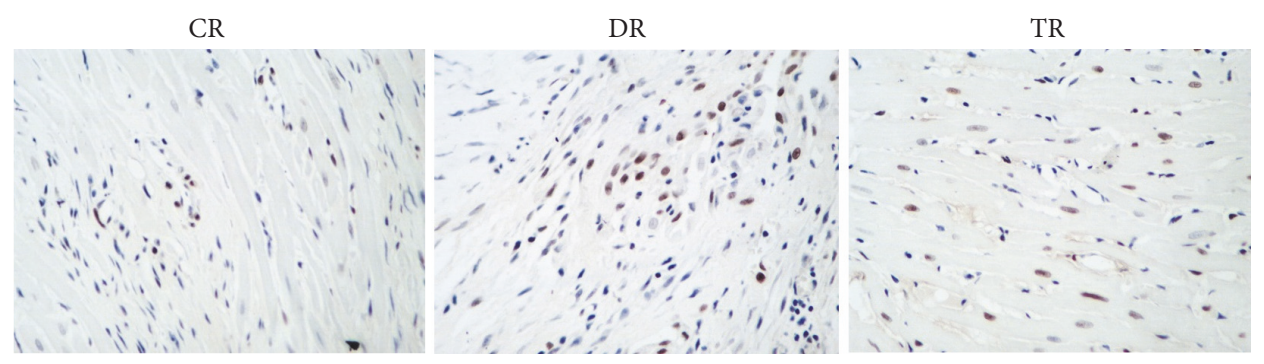

(h)

Figure 3: The expression of miR-503, Nrf2, downstream medium, and apoptosis level in streptozotocin- (STZ-) induced diabetes and treatment rats; CR: control rats; DR: diabetes rats; TR: treatment rats. (a) The expression of miR-503. (b) The gene expression of Nrf2. (c) The protein expression of Nrf2 and downstream medium. (d) The apoptosis level. (e) The apoptosis level by TUNEL staining (×200). *: compared with control rats, $P$ value $<0.05$ was considered to be statistically significant; \#: compared with diabetes rats, $P$ value $<0.05$ was considered to be statistically significant.

the ventricular remodeling appeared, in addition, in this process, the renin angiotensin aldosterone system (RAS) is activated, the cytokines is increased, and the fibroblast proliferation and myocardial fibrosis are promoted $[22,23]$. In this study, we found that myocardial structure and function were impaired and myocardial cell apoptosis level was increased in diabetes rats and these results were consistent with previous studies; model of DCM was successful. In addition, the impaired structure and function were reversed and the myocardial cell apoptosis level was decreased in diabetes rats after CPDT treatment. For CPDT, it has been reported to have a protective potential in neurogenic bladder cells and tissues [24-26]. In this study, for DCM, we also found that the CPDT had a protective potential; however, this mechanism remains unclear.

MicroRNAs have been reported to be involved in the pathogenesis of common human diseases, such as the nervous system diseases, cardiovascular system diseases, and cancer. Moreover, microRNAs have been reported to participate in the important physiological functions, such as autophagy, lysosome, autolysis, apoptosis, and cell cycle regulation [27]. In addition, microRNAs have also been reported 


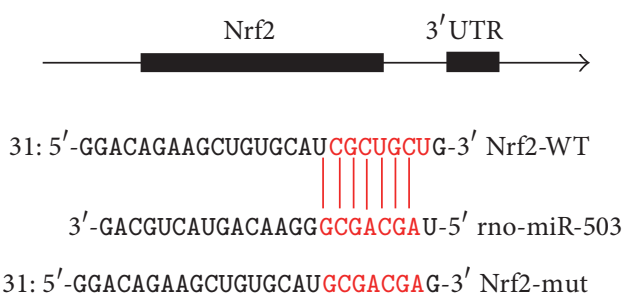

(a)

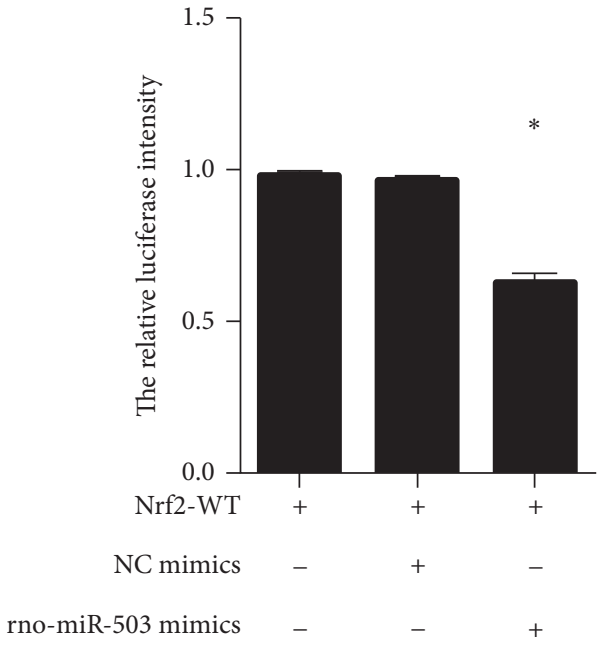

(b)

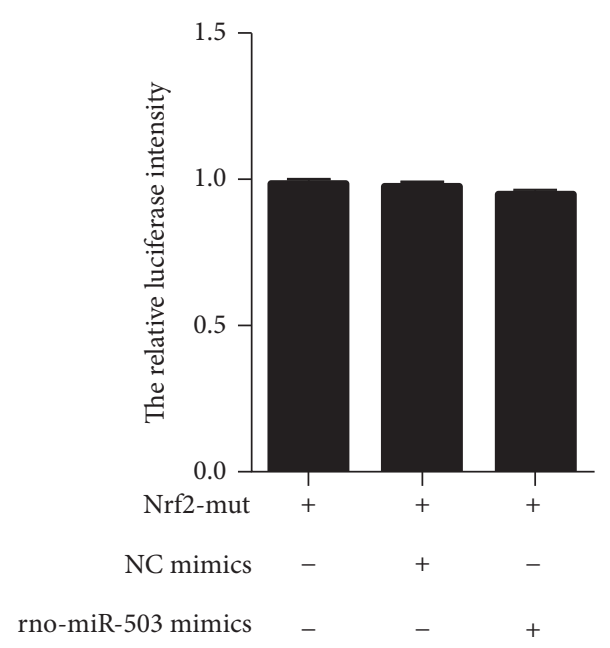

(c)

FIGURE 4: The Nrf2 was the target gene of miR-503; (a) putative miR-503 binding sequence in the wnt3a $3^{\prime}$-UTR and the site-directed mutant Nrf2 $3^{\prime}$-UTR. (b, c) The WT or mut reporter plasmids or NC- mimics (empty vector) or rno-miR-503 mimics (stable miR-503-overexpressing) were cotransfected into HEK293T cells. Relative repression of firefly luciferase expression was standardized to a transfection control. ${ }^{*} P<$ 0.05 .

to have ability of treating many diseases and microRNAs are potential to be a target for disease treatment [28]. Many studies have demonstrated that many abnormal microRNAs were involved in numerous important pathophysiological processes in DCM, such as myocardial hypertrophy, myocardial fibrosis, myocardial apoptosis, mitochondrial dysfunction, myocardial electrical remodeling, and epigenetic modification $[29,30]$. In this study, we found that miR-503 was also increased in DCM and closely correlated with occurrence and development of DCM. In addition, exhilaratingly, we also found that miR-503 was shown to be decreased in DCM treated by CPDT. Therefore, miR-503 was involved in DCM and CPDT exerted myocardial protection by performing miR-503.

To further investigate the downstream molecular mechanism of miR-503 in myocardial protection, the prediction analysis and dual-luciferase reporter assay method were used, and the results showed that nuclear factor erythroid 2related factor $2(\mathrm{Nrf} 2)$ had a putative miR-503-binding site mapped to the $3^{\prime}$-UTR and Nrf2 was the target gene of miR-503. Nrf2 is a novel transcription factor that belongs to the transcription factor Cap'n' Collar family, associated with the cytoplasmic chaperone Keapl in physiological state and plays important role in the regulation of oxidative stress response [31]. Under the source of oxidative stress, Nrf2 and
Keap1 solution is transferred into cell nucleus, combined with the antioxidant response element ARE locus, and the ARE was activated, playing an important role in protecting cells [32]. Therefore, Nrf2/ARE is one of the most important antioxidant pathways, playing an important role in the field of resistance to oxidation and chemical stimulation. Accumulating evidence has showed that a lot of oxygen free radicals were produced by oxidative stress in myocardial tissue, which can directly or indirectly damage the myocardium or affect the myocardial systolic and diastolic function and participate in the development of diabetic cardiomyopathy [33]. In addition, many oxygen free radical formations mediated myocardial cell apoptosis and also participate in the development of DCM [34]. In this study, we found that Nrf2 was decreased, myocardial apoptosis was increased in diabetes rats, and Phase II enzyme inducer (CPDT) had ability of upregulating expression of Nrf2 and reducing myocardial apoptosis in DCM. For CPDT, some studies have also showed that the protective function could depend on Nrf2/ARE pathway and CPDT could regulate the expression of Nrf2. Therefore, miR-503 is involved in the progress of DCM via regulating $\mathrm{Nrf} 2 / \mathrm{ARE}$ signaling pathway, $\mathrm{CPDT}$ reduces the development of DCM through miR-503 and Nrf2/ARE signaling pathway. In development of DCM, miR-503 was increased, Nrf2 was decreased, antioxidative stress ability was 


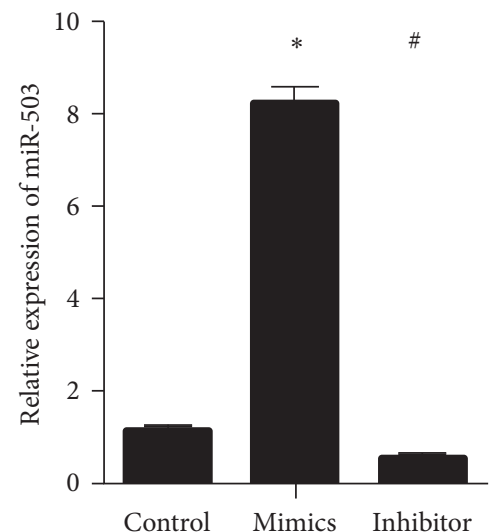

(a)

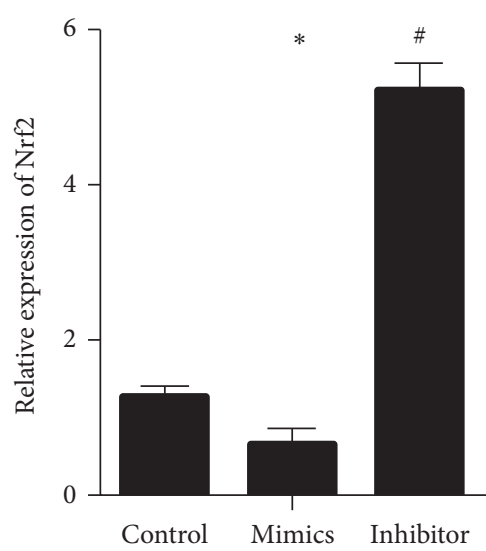

(b)

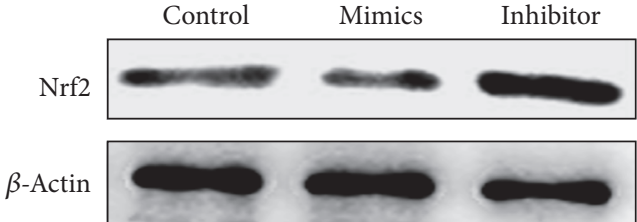

(c)

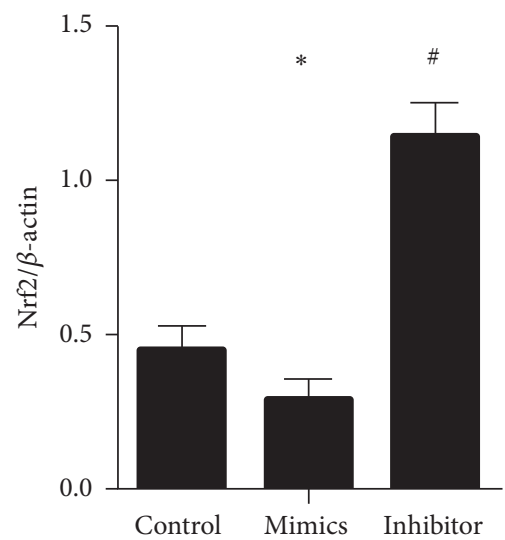

(d)

FIGURE 5: miR-503 regulated the expression of Nrf2 in myocardial cells; (a) the expression of miR-503; (b) the gene expression of Nrf2; (c-d) the protein expression of Nrf2; $*$ : compared with control, $P$ value $<0.05$ was considered to be statistically significant; \#: compared with mimics, $P$ value $<0.05$ was considered to be statistically significant.

weakened, and myocardial apoptosis was increased, damaging the myocardium or affecting the myocardial systolic and diastolic function. In treatment of CPDT for DCM, miR-503 was decreased, Nrf2 was increased, antioxidative stress ability was improved, and myocardial cell apoptosis was decreased, protecting the myocardium and improving the myocardial systolic and diastolic function.

In this study, we also investigated the downstream medium of Nrf2 in development of DCM and treatment of CPDT for DCM. We found that, in this progress, when Nrf2 was activated, malondialdehyde (MDA) and heme oxygenase 1 (HO-1) were also activated, and when Nrf2 was inhibited, MDA and HO-1 were also inhibited. The MDA is the final product of lipid peroxidation reaction of oxygen free radicals, attacks the unsaturated fatty acids, and plays an important role in the process of oxidative stress damage [35]. The HO-1 is a kind of microsomal oxidase with multiple functions and plays an important role in the process of oxidative stress [36]. These results also further confirmed that oxygen free radical attack occurs in DCM, and CPDT can reduce the content of oxygen free radicals and protect the myocardium.
In conclusion, miR-503 was involved in the progress of DCM via regulating Nrf2/ARE signaling pathway, and the CPDT reduces the occurrence and development of diabetic cardiomyopathy through miR-503 and Nrf2/ARE signaling pathway.

\section{Conflicts of Interest}

The authors declare no conflicts of interest.

\section{Authors' Contributions}

Ying Miao and Xiaoyu Liu contributed to study design, data collection, data analysis, and composition of the manuscript; Qin Wan conducted study design, data analysis, and composition of the manuscript; Yu Wang, Dan Liu, Honggang Zhou, and Nengbo Lin contributed to study design and data collection; Yi Luo contributed to study design, data collection, and data analysis; Jiyuan Zhong contributed to study design and data collection; all authors gave final approval of the version to be submitted. 


\section{References}

[1] C. Voulgari, D. Papadogiannis, and N. Tentolouris, "Diabetic cardiomyopathy: From the pathophysiology of the cardiac myocytes to current diagnosis and management strategies," Vascular Health and Risk Management, vol. 6, no. 1, pp. 883-903, 2010.

[2] L. Di Luigi, C. Corinaldesi, M. Colletti et al., "Phosphodiesterase type 5 inhibitor sildenafil decreases the proinflammatory chemokine CXCL10 in human cardiomyocytes and in subjects with diabetic cardiomyopathy," Inflammation, vol. 39, no. 3, pp. 1238-1252, 2016.

[3] Y. Kayama, U. Raaz, A. Jagger et al., "Diabetic cardiovascular disease induced by oxidative stress," International Journal of Molecular Sciences, vol. 16, no. 10, pp. 25234-25263, 2015.

[4] R. Ni, D. Zheng, and S. Xiong, "Mitochondrial calpain-1 disrupts ATP synthase and induces superoxide generation in type1 diabetic hearts: a novel mechanism contributing to diabetic cardiomyopathy," Diabetes, vol. 65, no. 1, pp. 255-268, 2016.

[5] R. Munday, Y. Zhang, C. M. Munday, and J. Li, "Structureactivity relationships in the induction of Phase II enzymes by derivatives of 3H-1,2-dithiole-3-thione in rats," ChemicoBiological Interactions, vol. 160, no. 2, pp. 115-122, 2006.

[6] L. Wang, M. Wang, J. Hu et al., "Protective effect of $3 \mathrm{H}-1$, 2-dithiole-3-thione on cellular model of Alzheimer's disease involves Nrf2/ARE signaling pathway," European Journal of Pharmacology, vol. 795, pp. 115-123, 2017.

[7] Z. Feng, Z. Liu, X. Li et al., " $\alpha$-Tocopherol is an effective Phase II enzyme inducer: Protective effects on acrolein-induced oxidative stress and mitochondrial dysfunction in human retinal pigment epithelial cells," The Journal of Nutritional Biochemistry, vol. 21, no. 12, pp. 1222-1231, 2010.

[8] J. E. Cohen, P. R. Lee, S. Chen, W. Li, and R. D. Fields, "MicroRNA regulation of homeostatic synaptic plasticity," Proceedings of the National Acadamy of Sciences of the United States of America, vol. 108, no. 28, pp. 11650-11655, 2011.

[9] J. Krol, I. Loedige, and W. Filipowicz, "The widespread regulation of microRNA biogenesis, function and decay," Nature Reviews Genetics, vol. 11, no. 9, pp. 597-610, 2010.

[10] P. Cai, T. Yang, X. Jiang, M. Zheng, G. Xu, and J. Xia, "Role of miR-15a in intervertebral disc degeneration through targeting MAP3K9," Biomedicine \& Pharmacotherapy, vol. 87, pp. 568574, 2017.

[11] R. G. Wallace, L. C. Twomey, M.-A. Custaud et al., "Potential Diagnostic and Prognostic Biomarkers of Epigenetic Drift within the Cardiovascular Compartment," BioMed Research International, vol. 2016, Article ID 2465763, 2016.

[12] X. H. Wang, R. Z. Qian, W. Zhang, S. F. Chen, H. M. Jin, and R. M. Hu, "MicroRNA-320 expression in myocardial microvascular endothelial cells and its relationship with insulin-like growth factor-1 in type 2 diabetic rats," Clinical and Experimental Pharmacology \& Physiology, vol. 36, no. 2, pp. 181-188, 2009.

[13] N. Pescador, M. Perez-Barba, J. M. Ibarra, A. Corbaton, M. T. Martinez-Larrad, and M. Serrano-Rios, "Serum circulating microRNA profiling for identification of potential type 2 diabetes and obesity biomarkers," PLOS ONE, vol. 8, no. 10, Article ID e77251, 2013.

[14] X. Li, N. Du, and Q. Zhang, "MicroRNA-30d regulates cardiomyocyte pyroptosis by directly targeting foxo3a in diabetic cardiomyopathy," Cell Death \& Disease, vol. 5, no. 10, Article ID e1479, 2014.
[15] M. Enomoto, T. Ishizu, Y. Seo et al., "Subendocardial systolic dysfunction in asymptomatic normotensive diabetic patients," Circulation Journal, vol. 79, no. 8, pp. 1749-1755, 2015.

[16] A. Hodzic, V. Ribault, P. Maragnes, P. Milliez, E. Saloux, and F. Labombarda, "Decreased regional left ventricular myocardial strain in type 1 diabetic children: a first sign of diabetic cardiomyopathy?" Journal of Translational Internal Medicine, vol. 4, no. 2, pp. 81-87, 2016.

[17] D. Suran, A. Sinkovic, and F. Naji, “Tissue Doppler imaging is a sensitive echocardiographic technique to detect subclinical systolic and diastolic dysfunction of both ventricles in type 1 diabetes mellitus," BMC Cardiovascular Disorders, vol. 16, no. 1, article no. 72, 2016.

[18] C. Drechsler, S. Kalim, J. B. Wenger et al., "Protein carbamylation is associated with heart failure and mortality in diabetic patients with end-stage renal disease," Kidney International, vol. 87, no. 6, pp. 1201-1208, 2015.

[19] M. Rajesh, S. Bátkai, M. Kechrid et al., "Cannabinoid 1 receptor promotes cardiac dysfunction, oxidative stress, inflammation, and fibrosis in diabetic cardiomyopathy," Diabetes, vol. 61, no. 3, pp. 716-727, 2012.

[20] M. Rajesh, P. Mukhopadhyay, S. Btkai et al., "Cannabidiol attenuates cardiac dysfunction, oxidative stress, fibrosis, and inflammatory and cell death signaling pathways in diabetic cardiomyopathy," Journal of the American College of Cardiology, vol. 56, no. 25, pp. 2115-2125, 2010.

[21] Z.-G. Ma, Y.-P. Yuan, S.-C. Xu et al., "CTRP3 attenuates cardiac dysfunction, inflammation, oxidative stress and cell death in diabetic cardiomyopathy in rats," Diabetologia, vol. 60, no. 6, pp. 1126-1137, 2017.

[22] Z. Li, C. S. Abdullah, and Z. Jin, "Inhibition of PKC- $\theta$ preserves cardiac function and reduces fibrosis in streptozotocin-induced diabetic cardiomyopathy," British Journal of Pharmacology, vol. 171, no. 11, pp. 2913-2924, 2014.

[23] V. Soetikno, F. R. Sari, V. Sukumaran et al., "Curcumin prevents diabetic cardiomyopathy in streptozotocin-induced diabetic rats: possible involvement of PKC-MAPK signaling pathway," European Journal of Pharmaceutical Sciences, vol. 47, no. 3, pp. 604-614, 2012

[24] X.-Y. Liu, C.-Y. Li, H. Bu et al., "The neuroprotective potential of phase II enzyme inducer on motor neuron survival in traumatic spinal cord injury in vitro," Cellular and Molecular Neurobiology, vol. 28, no. 5, pp. 769-779, 2008.

[25] X.-Y. Liu, C.-Y. Li, H. Bu et al., "Neuroprotective effect of CPDT on THA-induced cortical motor neuron death in an organotypic culture model," Brain Research Bulletin, vol. 83, no. 6, pp. 345-350, 2010.

[26] J. D. Paonessa, Y. Ding, K. L. Randall et al., "Identification of an unintended consequence of Nrf2-directed cytoprotection against a key tobacco carcinogen plus a counteracting chemopreventive intervention," Cancer Research, vol. 71, no. 11, pp. 3904-3911, 2011.

[27] P. Cong, S. Xiao, Y. Chen et al., "Integrated miRNA and mRNA transcriptomes of porcine alveolar macrophages (PAM cells) identifies strain-specific miRNA molecular signatures associated with H-PRRSV and N-PRRSV infection," Molecular Biology Reports, vol. 41, no. 9, pp. 5863-5875, 2014.

[28] M. P. LaPierre and M. Stoffel, "MicroRNAs as stress regulators in pancreatic beta cells and diabetes," Molecular Metabolism, vol. 6, no. 9, pp. 1010-1023, 2017. 
[29] A. Dorner, K. Schulze, U. Rauch, and H. P. Schultheiss, "Adenine nucleotide translocator in dilated cardiomyopathy: pathophysiological alterations in expression and function," Molecular and Cellular Biochemistry, vol. 174, pp. 261-269, 1997.

[30] X. Hu, T. Bai, Z. Xu, Q. Liu, Y. Zheng et al., "Pathophysiological fundamentals of diabetic cardiomyopathy," Comprehensive Physiology, vol. 7, pp. 693-711, 2017.

[31] K. Lu, A. L. Alcivar, J. Ma et al., "NRF2 Induction Supporting Breast Cancer Cell Survival Is Enabled by Oxidative Stress-Induced DPP3-KEAP1 Interaction," Cancer Research, vol. 77, no. 11, pp. 2881-2892, 2017.

[32] M. H. Lee, M. H. Han, D.-S. Lee et al., "Morin exerts cytoprotective effects against oxidative stress in $\mathrm{C} 2 \mathrm{C} 12$ myoblasts via the upregulation of Nrf2-dependent HO-1 expression and the activation of the ERK pathway," International Journal of Molecular Medicine, vol. 39, no. 2, pp. 399-406, 2017.

[33] Z. Xu, J. Sun, Q. Tong et al., "The role of ERK1/2 in the development of diabetic cardiomyopathy," International Journal of Molecular Sciences, vol. 17, no. 12, article no. 2001, 2016.

[34] P. Umbarkar, S. Singh, S. Arkat, S. L. Bodhankar, S. Lohidasan, and S. L. Sitasawad, "Monoamine oxidase-A is an important source of oxidative stress and promotes cardiac dysfunction, apoptosis, and fibrosis in diabetic cardiomyopathy," Free Radical Biology \& Medicine, vol. 87, pp. 263-273, 2015.

[35] Y. Zhu, Y.-J. Zhang, W.-W. Liu, A.-W. Shi, and N. Gu, "Salidroside suppresses HUVECs cell injury induced by oxidative stress through activating the Nrf2 signaling pathway," Molecules, vol. 21, no. 8, article no. 1033, 2016.

[36] Y. C. Hseu, C. W. Chou, K. J. Senthil Kumar et al., "Ellagic acid protects human keratinocyte (HaCaT) cells against UVAinduced oxidative stress and apoptosis through the upregulation of the HO-1 and Nrf-2 antioxidant genes," Food and Chemical Toxicology, vol. 50, no. 5, pp. 1245-1255, 2012. 


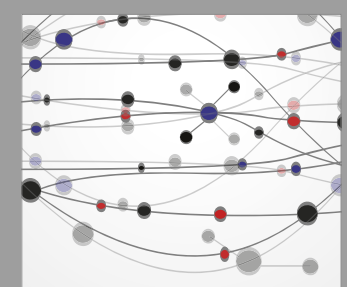

The Scientific World Journal
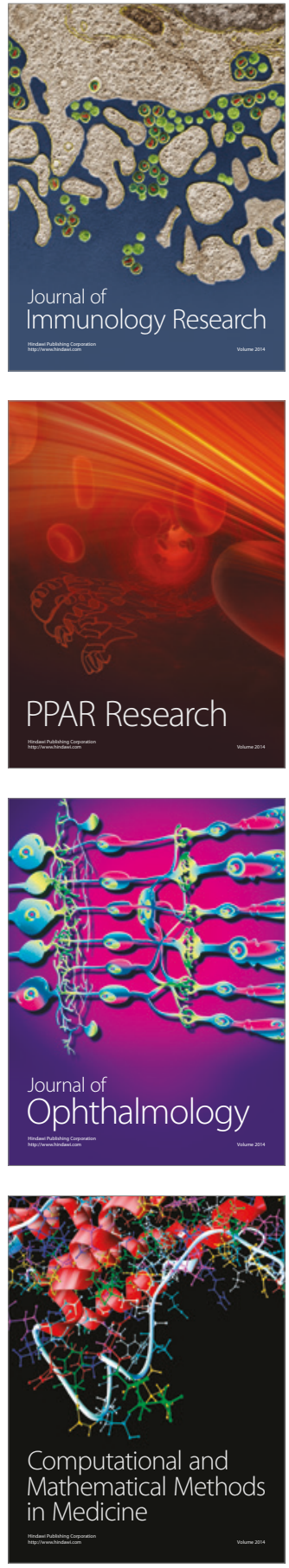

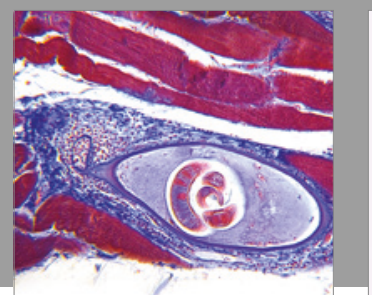

Gastroenterology Research and Practice
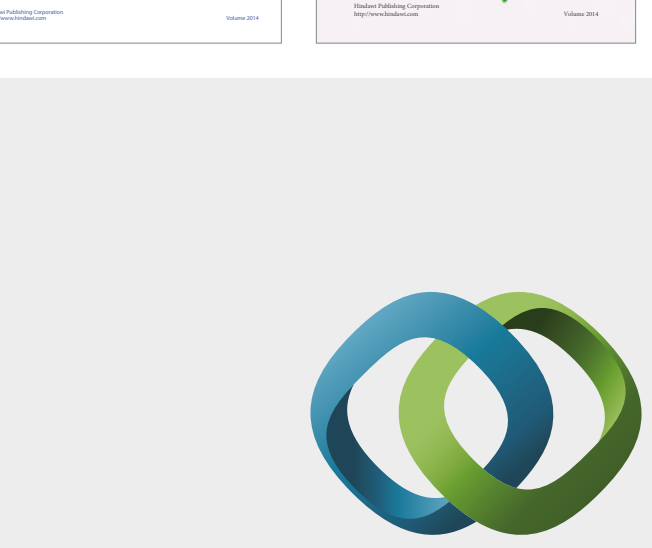

\section{Hindawi}

Submit your manuscripts at

https://www.hindawi.com
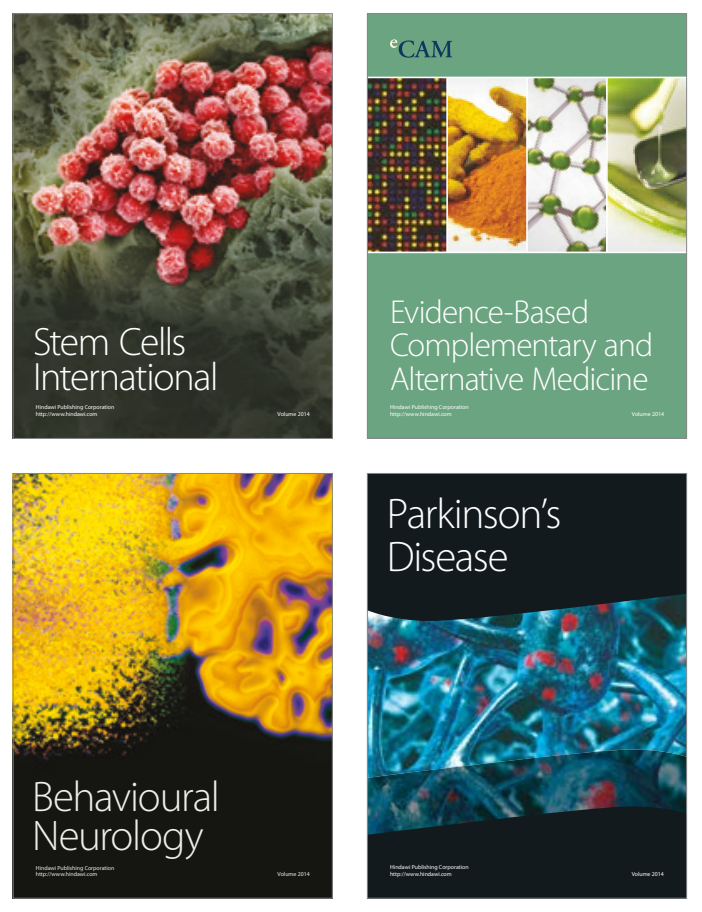
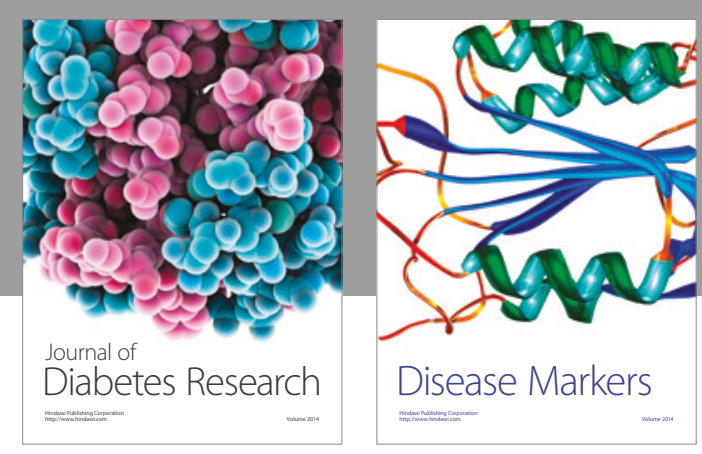

Disease Markers
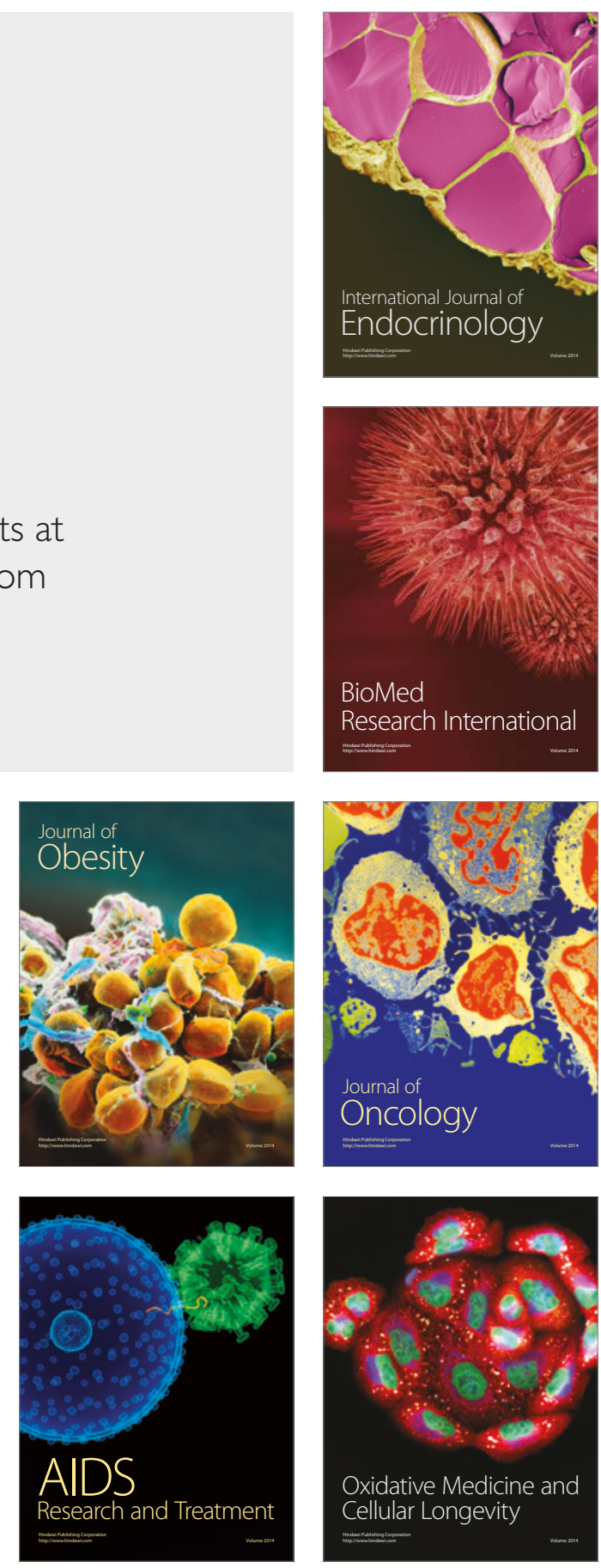